\title{
Conflitos na relação entre Cirurgiões-dentistas e operadoras de Odontologia suplementar
}

\author{
Conflicts in the Relationship Between Dentists and Health \\ Insurance Providers
}

\author{
Larissa Barradas Lima Feitosa ${ }^{1}$, Denise Ribeiro Santos ${ }^{2}$, \\ Rai Matheus Carvalho Santos ${ }^{3}$
}

DOI: http://dx.doi.org/10.11606/issn.2317-2770.v25i1p15-22

\begin{abstract}
Feitosa LBL, Santos DR, Santos RMC. Conflitos na relação entre Cirurgiões-dentistas e operadoras de Odontologia suplementar. Saúde, Ética \& Justiça. 2020;25(1):15-22.

RESUMO: Este artigo buscou levantar na literatura nacional os motivos pelos quais a relação entre as operadoras de planos de saúde e os cirurgiões-dentistas é conflituosa, traçando um histórico do mercado brasileiro na área, bem como o perfil do profissional deste segmento, destacando as implicações observadas quanto à autonomia profissional, remuneração e satisfação com o trabalho, a fim de fomentar uma reflexão crítica na categoria quanto às relações de trabalho no âmbito da assistência odontológica suplementar. A análise realizada evidenciou que o sistema de saúde suplementar se desenvolveu por mais de trinta anos sem interferência governamental, culminando em um crescimento alarmante, desordenado e desregulado, influenciado pelo excesso de oferta de dentistas e redução de pacientes que pagam por serviços privados. Dessa forma, grande parcela dos profissionais que têm se associado a planos de saúde na expectativa de expansão dos seus serviços são submetidos às demandas das operadoras que limitam seu trabalho àquele menos oneroso, interferem no plano de tratamento e utilizam tabelas de preços desatualizadas para o pagamento de procedimentos clínicos, levando a uma percepção negativa e descontentamento em relação ao desempenho das operadoras. Concluiu-se que de fato a relação é conflituosa, atingindo o profissional em diversas esferas, não apenas econômicas como pessoais, e está associada à expansão do sistema suplementar. Sendo assim, a prática odontológica é afetada na sua essência, sem que medidas de controle e supervisão, tão necessárias, sejam implementadas para corrigir assimetrias nas relações corporativas e evitar condições precárias da profissão.
\end{abstract}

DESCRITORES: Saúde Suplementar; Assistência Odontológica; Planos de Pré-Pagamento em Saúde.

\footnotetext{
${ }^{1 .}$ UNICEPLAC - Centro Universtário do Planalto Central Apparecido dos Santos. E-mail: laarissarlf@gmail.com

2. Universidade de Brasília. E-mail: deniseribeiro.s93@gmail.com

3. UNICEPLAC - Centro Universitário do Planalto Central Apparecido dos Santos. E-mail: rai.santos@uniceplac.edu.br

Endereço para correspondência: rai.santos@uniceplac.edu.br
} 


\section{INTRODUÇÃO}

A odontologia no Brasil, historicamente, sempre teve sua prática centrada na clínica privada. Entretanto, o aumento do número de cirurgiões-dentistas e a diminuição do desembolso direto dos pacientes para custear os tratamentos levou à crise do modelo liberal ${ }^{1,2,3}$. Por outro lado, a demanda elevada de usuários com necessidades odontológicas e a inacessibilidade de grande parte da população aos serviços públicos de saúde contribuíram para a expansão das operadoras de odontologia suplementar ${ }^{4}$. Frente a essa situação, os profissionais têm buscado associação (vinculação) aos convênios e credenciamentos como estratégia contra a crise e na tentativa de permanecer no mercado ${ }^{1}$. No entanto se deparam com as operadoras interferindo no seu processo de trabalho e experimentam uma queda progressiva no poder de exercício da profissão como atividade autônoma ${ }^{3,5}$.

Nesse contexto, o profissional é o detentor da força de trabalho, mas está submetido às imposições de um mercado regido pela lei da oferta e procura e de mais-valia, as quais limitam a sua liberdade de atuação profissional, principalmente relativa ao controle do preço de seu trabalho ${ }^{6,7}$, transformando o cirurgião-dentista em um "empregado" sem direitos trabalhistas, remunerado exclusivamente segundo a sua produção $0^{8,9,10}$.

Em 2002, o Conselho Federal de Odontologia (CFO) publicou no Diário Oficial da União (DOU) uma tabela com os valores de referência para procedimentos odontológicos (VRPO), com o objetivo de orientar a remuneração mínima dos cirurgiões-dentistas ${ }^{11,12,13}$. No entanto, estudos relatam que muitos convênios utilizam tabelas próprias para a remuneração de seus credenciados ${ }^{14}$, o que leva muitos profissionais a aumentar a sua carga horária de trabalho para suprir a defasagem dos honorários que são pagos pelos planos $^{15}$, uma condição que pode configurar aviltamento profissional, previsto como uma infração ao artigo 21 do Código de Ética Odontológica ${ }^{16,17}$.

A insatisfação com a tabela de honorários paga pelas operadoras foi evidenciada por diversos autores ${ }^{18-21}$. Uma pesquisa avaliou o grau de satisfação de cirurgiõesdentistas credenciados a planos odontológicos no estado de São Paulo e constatou que $70 \%$ dos profissionais classificaram como ruim/péssima a atuação das operadoras dos planos de saúde ${ }^{15}$. Além da insatisfação com o pagamento de honorários, outros estudos observaram a existência de problemas relacionados à glosa e não liberação de procedimentos ${ }^{7}$, o que gera tensão entre a autonomia técnica do profissional e a relação contratual estabelecida entre as partes ${ }^{23,3}$.

Entender as assimetrias que existem nas relações profissionais e que levam à precarização do trabalho dos profissionais de saúde, com reflexos na população, possibilita avaliar o dinamismo e as contradições da realidade social na qual os cirurgiões-dentistas estão inseridos $^{23,7}$. Diante desse contexto, este artigo buscou analisar os conflitos existentes na relação entre cirurgiõesdentistas e operadoras de odontologia suplementar, a partir dos estudos disponíveis na literatura científica, destacando a vulnerabilidade e precarização do trabalho destes profissionais no setor.

\section{MÉTODO}

Trata-se de revisão de literatura que surgiu a partir da seguinte questão norteadora: "Quais os conflitos entre as operadoras de odontologia suplementar e os cirurgiões-dentistas?". Para responder a esta pergunta, foram realizadas buscas nas seguintes bases de dados nacionais e internacionais: Medical Literature Analysis and Retrieval System Online (MEDLINE), Literatura Latino-Americana em Ciências de Saúde (LILACS), Scientific Electronic Library Online (SciELO) e Biblioteca Brasileira de Odontologia (BBO), indexados na Biblioteca Virtual da Saúde (BVS). Para a seleção dos artigos foram utilizados os seguintes critérios de inclusão: textos completos com livre acesso a bases de dados nos idiomas português, inglês e/ou espanhol, que discorressem sobre conflitos entre operadoras de odontologia suplementar e cirurgiões-dentistas. Devido à escassez de estudos, foi estabelecido como limite temporal os estudos publicados nos últimos 20 anos. Para a seleção dos artigos foram considerados os seguintes descritores: "saúde suplementar" ("supplementary health"), "assistência odontológica" ("dental care") e "planos de saúde" ("prepaid health plan"). Em um primeiro momento, os manuscritos foram selecionados pelo título e resumo, sendo, posteriormente, avaliados na íntegra para uma completa apreciação do material retido.

Os artigos foram organizados em três grandes áreas de acordo com a matéria abordada: expansão do mercado da odontologia suplementar no Brasil, perfil do profissional credenciado brasileiro e dados relativos ao exercício profissional em saúde suplementar, como autonomia e satisfação econômica e profissional.

\section{DESENVOLVIMENTO}

\section{Expansão das operadoras de odontologia suplementar e a inserção do cirurgião-dentista}

A Constituição de 1988 foi importante para a legitimação da saúde como um dos direitos sociais ${ }^{24} \mathrm{e}$ para a criação do sistema público de saúde brasileiro, representado pelo Sistema Único de Saúde (SUS), cujo processo de construção apresentou importantes mecanismos de regulação, tais como a Lei Orgânica da Saúde $n^{\circ} 8.080 / 90^{25}$, a extinção do Instituto Nacional de 
Assistência Médica da Previdência Social (INAMPS) ${ }^{26}$, a Norma Operacional Básica (NOB)/9327, a (NOB)/96 28 e a implantação do Piso da Atenção Básica (PAB) em $1998^{29}$. Como se observa, o sistema de saúde público experimentou importantes marcos regulatórios ao longo do seu processo de construção, no entanto, não se pode dizer o mesmo do setor de saúde suplementar, que durante mais de trinta anos sem a interferência governamental, culminou em um crescimento alarmante, desordenado e desregulado ${ }^{30}$.

$\mathrm{Na}$ década de 90, foram criados órgãos e mecanismos de proteção ao usuário e consumidor, tais como o Código de Defesa do Consumidor (CDC) $)^{31}$ e o Programa de Proteção e Defesa do Consumidor $(\text { PROCON })^{32}$, que atuam principalmente diante de conflitos envolvendo consumidores e operadoras de planos de saúde ${ }^{33}$. Somente em 1998, ou seja, dez anos após a promulgação da Constituição Federal (CF) que estabeleceu a criação do SUS, o setor saúde suplementar deu início a uma legislação inerente de regulação, com a aprovação da Lei $n^{\circ} 9.656^{34}$, que dispõe sobre os planos e seguros privados de assistência à saúde, e no ano de 2000, com a Lei $n^{\circ} 9.961$ que concebeu a Agência Nacional de Saúde Suplementar (ANS) ${ }^{35,36}$.

Após a promulgação dos mecanismos de regulação, observou-se aumento no número de usuários de planos odontológicos, que passou de cerca de 3 milhões no ano de 2001, para aproximadamente 22 milhões no ano de $2016^{2,37,38,39}$. O crescimento vertiginoso do setor de saúde suplementar se deu num cenário em que acontecia o fenômeno inverso: crise do modelo liberal de prestação de serviços odontológicos ${ }^{1,2}$, historicamente centrado nas clínicas privadas ${ }^{40}$. Entre os fatores associados à instabilidade no mercado de trabalho privado na área da odontologia, pode-se destacar o excesso de oferta de cirurgiões-dentistas, que pode ter sido influenciado por distorções no mercado educacional ${ }^{8}$, conglomeração dos serviços nos centros urbanos brasileiros ${ }^{29,41}$ e queda de desembolso direto dos pacientes para financiar os tratamentos ${ }^{1}$.

Nesse contexto, os cirurgiões-dentistas passaram a enxergar o convênio com as operadoras de odontologia suplementar como estratégia para expandir o comércio, uma vez que proporciona o acesso a um mercado consumidor amplo. Este é composto por pacientes que se sentem atraídos por condições mais acessíveis quanto ao custo-benefício apresentado pelos planos de saúde $^{42}$, aumentando, por sua vez, a competitividade e estabilidade do profissional na dinâmica do mercado de trabalho ${ }^{1}$.

Essa mudança na conformação da assistência odontológica privada refletiu-se na maior adesão dos cirurgiões-dentistas aos convênios e credenciamentos, como pôde ser observado em estudos realizados pelo Conselho Federal de Odontologia, o qual verificou que aproximadamente metade dos profissionais inscritos no conselho prestava serviços em conjunto a convênios ${ }^{17}$. Outro estudo transversal do tipo inquérito desenvolvido em 2017, com 108 cirurgiões-dentistas no Distrito Federal, observou o predomínio dos atendimentos odontológicos em consultórios particulares intermediados por planos de saúde e a quase inexistência da modalidade de desembolso direto ${ }^{17}$.

\section{Perfil do cirurgião-dentista credenciado a operadoras de odontologia suplementar}

Um estudo realizado em 2004, que entrevistou 150 cirurgiões-dentistas, constatou que $56,6 \%$ eram do sexo masculino, trabalhavam de 7 a 9 horas por dia no consultório e identificou a Uniodonto como a empresa mais solicitada pelos profissionais, quando comparada a outras operadoras de assistência odontológica ${ }^{43}$. Quanto à faixa etária, Vilela et al. ${ }^{44}$ observaram que entre os 500 cirurgiões-dentistas entrevistados na Região Metropolitana do Vale do Paraíba, Litoral Norte, Alto Tietê e Baixa Mogiana no estado de São Paulo, 90\% tinham menos de 40 anos de idade e $77 \%$ até 10 anos de formados. Outro achado semelhante foi encontrado por estudo transversal realizado na cidade de Araraquara, no interior do estado de São Paulo, onde se observou a prevalência de 53,2\% dos entrevistados com até 10 anos de formados ${ }^{43}$.

Com relação à distribuição por regiões do país, uma pesquisa realizada em 2003 pela Associação Brasileira de Odontologia (ABO), Federação Interestadual dos Odontologistas (FIO), Conselho Federal de Odontologia (CFO) e Federação Nacional dos Odontologistas (FNO), verificou que $48 \%$ dos cirurgiões-dentistas exerciam seu trabalho associados aos convênios, principalmente na região $\mathrm{Sul}(60,7 \%)$, enquanto que nas outras regiões este percentual foi de apenas $14,7 \%{ }^{45}$.

Em um único trabalho em que se avaliou a especialização do profissional que presta serviço à convênios, encontrou-se que $60 \%$ relataram possuir alguma especialização. Também nesse mesmo estudo, observou-se que $50 \%$ dos profissionais declararam trabalhar menos de 5 anos associados a operadoras e apenas $38 \%$ mais de 5 anos, o que pode indicar que muitos profissionais enxergam o convênio como um trabalho provisório e que pode contribuir para a melhoria das habilidades no que se refere ao exercício da profissão ${ }^{44}$.

Em uma pesquisa sobre auditoria odontológica realizada em município do estado do Rio de Janeiro verificou-se, por meio da análise de 2.283 relatórios de avaliações clínicas iniciais, que os procedimentos de dentística $(50,1 \%)$ e periodontia $(19,4 \%)$ foram responsáveis pela maior parte das glosas, enquanto as especialidades de cirurgia oral menor e endodontia 
corresponderam a apenas $2,2 \%$ e $2,3 \%$ das glosas, respectivamente ${ }^{46}$.

\section{Autonomia profissional, remuneração e grau de satisfação}

A adesão a convênios e credenciamentos tem impactado o exercício da profissão pelos cirurgiõesdentistas. As operadoras, ao definirem regras de conduta procedimental e valorarem os procedimentos odontológicos prestados frente a critérios próprios, acabam por limitar o trabalho do profissional ao tratamento menos oneroso e, portanto, mais lucrativo ${ }^{7}$, reforçando a ideia da tomada de decisão em uma relação "patrão e empregado" e, consequentemente, terceirizando a força de trabalho do cirurgião-dentista ${ }^{47}$. O comprometimento da autonomia do profissional para decidir e executar serviços ${ }^{7}$ posiciona as operadoras no controle econômico da prestação de serviço ofertada ${ }^{2,43,48,49}$.

Estudos anteriores observaram que os cirurgiõesdentistas afirmaram sofrer interferências na oferta do plano de tratamento necessário aos pacientes. Há relatos de realização de manobras comerciais de determinadas operadoras para obter vantagem econômica através da não-autorização sistemática da realização de procedimentos, que, muitas vezes, chegam a prescrever ${ }^{7,47,50,51}$. Esse cenário favorece a prática de infração ética na prestação de serviços odontológicos, tendo em vista que, de acordo com o artigo $5^{\circ}$ do Código de Ética Odontológica $(2012)^{52}$, é direito do cirurgiãodentista garantir a saúde bucal ao paciente de forma integral por meios de conhecimento técnico e específico, conforme os princípios da beneficência. Ainda, de acordo com o artigo $3^{\circ}$ do mesmo código, cabe aos profissionais da Odontologia dirigir ações com o objetivo de promover a saúde do ser humano e que visem a satisfazer as necessidades de saúde da população e para garantir a integralidade da assistência à saúde e a preservação da autonomia dos indivíduos.

Além das tensões no que se refere à autonomia técnica dos profissionais, o descontentamento com os valores pagos pelos procedimentos odontológicos é outro motivo para conflitos entre prestadores e operadoras ${ }^{3}$. Um estudo realizado em um município do interior do estado de São Paulo verificou que os profissionais classificaram como regular ou ruim os valores dos procedimentos clínicos repassados pelas operadoras ${ }^{23}$. De acordo o Sindicato Nacional das Empresas de Odontologia de Grupo $(\mathrm{SINOG})^{53}$, além da grande concorrência de mercado, as operadoras também enfrentam como desafios a crise econômica do país e a elevada carga tributária em decorrência do complexo sistema de arrecadação brasileiro, o que impacta nas tabelas de preço utilizadas, fazendo com que o cirurgião-dentista conveniado necessite atender cerca de sete a dez pacientes para receber honorário de um paciente particular, atendido por um profissional liberal plenamente estabelecido no mercado de trabalho ${ }^{54,55}$.

Tais achados sugerem a precarização do trabalho executado pelo prestador de saúde conveniado, refém em meio à guerra no mercado odontológico suplementar, caracterizado pela atuação de muitas operadoras que não abrem mão de maximizar o lucro sobre a mão de obra de demanda complexa do ponto de vista científico e ocupacional ${ }^{46}$. Essa postura mercadológica e o controle do valor da troca comercial pelas operadoras de saúde suplementar ${ }^{1,56}$ influenciam negativamente a percepção dos profissionais a respeito dos convênios e credenciamentos, conforme foi observado por um estudo realizado em 2013 na cidade de São Paulo, o qual verificou que não só os cirurgiões-dentistas, mas também médicos e fisioterapeutas possuem uma relação e uma visão negativa quando se trata de saúde suplementar ${ }^{15}$. A insatisfação dos cirurgiões-dentistas credenciados a planos odontológicos foi também observada em estudos realizados no estado da Paraíba ${ }^{19}$, Santa Catarina ${ }^{20}$ e Rio de Janeiro ${ }^{18}$.

Outros fatores que também foram citados na literatura no sentido de reduzir o grau de satisfação dos cirurgiões-dentistas conveniados seriam a relação inflexível de determinadas empresas, a falta de informação fornecida ao paciente ${ }^{7}$ e a burocracia para a autorização de procedimentos e repasse financeiro ${ }^{23,57}$. Importante destacar, também, a ausência de preocupação e investimento das empresas na formação continuada de seus profissionais, tendo em vista que um estudo realizado em 2012 observou que $63 \%$ dos cirurgiões-dentistas entrevistados relataram não ter participado de palestras ou não ter recebido periódicos para a atualização dos conhecimentos específicos da área ${ }^{57}$.

\section{DISCUSSÃO E CONCLUSÕES}

O crescimento do setor de saúde suplementar, influenciado pelas limitações da assistência odontológica no setor público e pela crise do modelo liberal autônomo, tem afetado a prática odontológica ao atrair um número cada vez maior de cirurgiões-dentistas que necessitam garantir sua sobrevivência em um mercado de trabalho saturado e que, portanto, enxergam no convênio uma estratégia para expandir seus serviços. Dessa forma, tem se observado que muitas operadoras, ao comercializar a assistência odontológica, visando a maximização do lucro em detrimento da prestação de serviço de qualidade e de valorização e respeito ao capital humano, utilizam mecanismos que impõem valores de ressarcimento inadequados e reduzem a autonomia ético-tecnológica do profissional, corroborando para a terceirização da sua força de trabalho e para condições de precarização da profissão. Estes achados alertam para a necessidade de 
rediscutir o modelo de atenção suplementar e elaborar medidas de controle e fiscalização das faculdades de odontologia e das operadoras de saúde suplementar.

Nesse sentido, caberia uma maior atuação do Conselho Federal de Odontologia junto aos Conselhos Regionais de Odontologia visando corrigir assimetrias nas relações corporativas para proteger os cirurgiões- dentistas de situações que caracterizem precarização do trabalho. Tais medidas poderiam também proporcionar uma adequada assistência à saúde no âmbito suplementar para uma grande parcela da população desassistida pelo sistema público de saúde brasileiro e, portanto, contribuir para a inclusão social dos vulneráveis e para a dignidade humana.

Feitosa LBL, Santos DR, Santos RMC. Conflicts in the Relationship Between Dentists and Health Insurance Providers. Saúde, Ética \& Justiça. 2020;25(1):15-22.

\begin{abstract}
This article aimed to find, in the national literature, the reasons for the conflictive relationship between health insurance providers and dental surgeons. The study describes the history of insurance companies in the Brazilian market and the professional profile of dental surgeons. Issues related to the autonomy, remuneration and job satisfaction of the health care professional were found. This might encourage critical thought among these professionals regarding work relations in the field of supplementary dental care. The analysis showed that the supplementary health system developed for over thirty years without government interference, culminating in an alarming, disorderly and unregulated growth, influenced by the excess supply of dentists and the reduction in the number of patients who pay for private services. Professionals who contract with health insurance companies in the hopes of expanding their practice must yield to their demands. These companies often limit the provider to less costly treatment, interfere with their treatment plan and use outdated price lists to pay for clinical procedures, leading to a negative perception and dissatisfaction with the insurance companies' performance. The studied relationship was found to be truly conflictive, affecting the healthcare provider not only at the financial but also personal level. This conflict is related to the growth of the supplementary health system, which affects dental practice at its core, without the due control and supervision measures, necessary to correct asymmetries in corporate relations and avoid precarious working conditions, being taken.
\end{abstract}

KEY WORDS: Supplemental Health; Dental Care; Prepaid Health Plans.

\title{
REFERENCIAS
}

1. Vieira C, Costa NR. Estratégia profissional e mimetismo empresarial: os planos de saúde odontológicos no Brasil. Ciênc Saúde Coletiva [Internet]. 2008 [Acesso em 2019 dez. 10];13(5):1579-88. Disponível em: http://www. scielo.br/scielo.php?script $=$ sci_arttext\&pid $=\mathrm{S} 1413$ $81232008000500022 \& \operatorname{lng}=$ en. $\quad$ DOI: https://doi. org/10.1590/S1413-81232008000500022

2. Garbin D, Mattevi GS, Carcereri DL, Caetano JC. Odontologia e saúde suplementar: marco regulatório, políticas de promoção da saúde e qualidade da atenção. Ciênc Saúde Coletiva [Internet]. 2013 [Acesso em 2019 dez. 10];18(2):441-52. Disponível em: http://www. scielo.br/scielo.php?script=sci_arttext\&pid=S1413$81232013000200015 \& \operatorname{lng}=$ en. $\quad$ DOI: http://dx.doi. org/10.1590/S1413-81232013000200015

3. Neumann DG, Finkler M, Caetano JC. Relações e conflitos no âmbito da saúde suplementar: análise a partir das operadoras de planos odontológicos. Physis [Internet]. 2017 [Acesso em 2019 dez. 10];27(3):453-74. Disponível em: $\quad$ http://www.scielo.br/scielo.php?script=sci arttext\&pid=S0103-73312017000300453\&lng=en. DOI: https://doi.org/10.1590/s0103-73312017000300005

4. Brasil. Ministério da Saúde. Agência Nacional de Saúde Suplementar (ANS). Caderno de Informação da Saúde Suplementar: beneficiários, operadoras e planos. Rio de Janeiro: ANS; 2010.
5. Neumann DG. Modelo de atenção em saúde bucal na saúde suplementar: uma análise a partir das operadoras de planos odontológicos no Brasil [tese] [Internet]. Florianópolis: Universidade Federal de Santa Catarina; 2014. [Acesso em 2019 dez. 10]. Disponível em: https://repositorio.ufsc. br/xmlui/bitstream/handle/123456789/129479/327517. pdf? sequence $=1 \&$ isAllowed $=y$

6. Portillo JAC. Saúde bucal e o mercado de trabalho odontológico. Rev Saúde em Debate. 1986;18:52-64.

7. Moraes DA, Maluf F, Tauil PL, Portillo JAC. Precarização do trabalho odontológico na saúde suplementar: uma análise bioética. Ciênc Saúde Coletiva [Internet]. 2019 [Acesso em 2019 nov. 10];24(3):705-14. Disponível em: http:// www.scielo.br/scielo.php?script=sci_arttext\&pid=S141381232019000300705\&lng=en. $\quad$ DOI: https://doi. org/10.1590/1413-81232018243.01972017

8. Zanetti CHG. A crise da odontologia brasileira: as mudanças estruturais do mercado de serviços e o esgotamento do modo de regulação curativo de massa. Revista Ação Coletiva. 1999;2(3):11-24.

9. Brasil. Agência Nacional de Saúde Suplementar (ANS). Regulação e Saúde: Planos Odontológicos: uma abordagem econômica no contexto regulatório. Rio de Janeiro: Ministério da Saúde; 2002.

10. Barbosa GMS. Obrigatoriedade da odontologia nos planos 
Feitosa LBL, Santos DR, Santos RMC. Conflitos na relação entre Cirurgiões-dentistas e operadoras de Odontologia suplementar.

de referência (ampliação da oferta?). Projeto apresentado ao Ministério da Saúde, Agência Nacional de Saúde Suplementar; 2003. p.19.

11. Conselho Federal de Odontologia (CFO). Tabela de valores referenciais para convênios e credenciamentos. Diário Oficial da União. 2002 jan 24.

12. Maruo IT, Saga A, Camargo ES, Guariza Filho O, Tanaka $\mathrm{O}$, Maruo H. Valores Referenciais para Procedimentos Odontológicos (VRPO) em ortodontia. Rev Dent Press Ortodon Ortop Facial [Internet]. 2009 [Acesso em 2019 dez. 10];14(3):40-3. Disponível em: http://www. scielo.br/scielo.php?script $=$ sci_arttext\&pid $=\mathrm{S} 1415$ 54192009000300006\&lng=en. $\quad$ DOI: https://doi. org/10.1590/S1415-54192009000300006

13. Zimmermann RD, De Paula FJ, Silva M. Honorários profissionais do cirurgião-dentista. In: Zimmermann RD, De Paula FJ, Silva M. Deontologia odontológica: ética e legislação. $1^{\text {a }}$ ed. São Paulo: Santos; 2011. p.193-208.

14. Lemos JA, Wanderley MS, Moreira PVL, Rabello PM, Caldas JR. AF, Rosenblatt A. Estudo comparativo entre convênios odontológicos, empresas e demais entidades prestadoras e/ou contratantes de serviços odontológicos na cidade de João Pessoa/PB. Rev Fac Odontol Pernamb. 1997;15:73-6.

15. Agência Brasil. Maioria dos médicos de São Paulo considera atuação dos planos de saúde regular ou ruim [Internet]. São Paulo; 2013. [Acesso em 10 nov. 2019]. Disponível em: https://www.olhardireto.com.br/noticias/ exibir.asp?id $=317469$ \&noticia $=$ maioria-dosmedicos-desao-paulo-considera-atuacao-dos-planos-de-saude-regularou-ruim

16. Santos RB, Ciuffi F. Dos honorários profissionais. In: Santos RB, Ciuffi F. Aspectos éticos e legais da prática odontológica: código de ética odontológica comentado. $1^{\mathrm{a} e d . ~ S a ̃ o ~ P a u l o: ~ S a n t o s ; ~ 2009 . ~ p .67-75 . ~}$

17. Conselho Federal de Odontologia (CFO). Código de ética odontológica [online]. Brasília (DF); 2009. [Acesso em 16 out. 2019]. Disponível em: https://website.cfo.org.br/wpcontent/uploads/2018/03/codigo_etica.pdf

18. Matos IB. Expectativas do exercício profissional de graduados em odontologia [tese] [Internet]. Rio de Janeiro: Escola Nacional de Saúde Pública Sérgio Arouca, Fundação Oswaldo Cruz; 2005. [Acesso em 2019 nov. 20]. Disponível em: https://www.arca.fiocruz.br/handle/icict/4584

19. D'Ávila S, Oliveira PAP, Lucas RSCC, Souza EA. Assistência odontológica x plano de saúde: um estudo em Campina Grande, Paraíba, Brasil. Pesq Bras Odontoped Clin Integr. 2007;7(3):259-63. DOI: https://doi.org/10.403 $4 / 1519.0501 .2007 .0073 .0011$

20. Pietrobon L. Planos de saúde: uma análise das relações entre as operadoras, prestadores de serviço e beneficiários sob a visão do cirurgião-dentista [tese]. [Internet]. Florianópolis: Centro de Ciências da Saúde, Universidade Federal de Santa Catarina; 2010. [Acesso em 2019 nov. 20]. Disponível em: http://repositorio.ufsc.br/handle/123456789/103310

21. Garbin D, Mattevi GS, Carcereri DL, Caetano JC. Odontologia e saúde suplementar: marco regulatório, políticas de promoção da saúde e qualidade da atenção.
Ciênc Saúde Coletiva [Internet]. 2013 [Acesso em 2019 nov. 10];18(2):441-52. Disponível em: http://www. scielo.br/scielo.php?script=sci_arttext\&pid=S141381232013000200015\&lng=en. $\quad$ DOI: https://doi. org/10.1590/S1413-81232013000200015

22. Daros RF, Gomes RS, Silva FH, Lopes TC. A satisfação do beneficiário da saúde suplementar sob a perspectiva da qualidade e integralidade. Physis [Internet]. 2016 [Acesso em 2019 nov. 10];26(2):525-47. Disponível em: http:// www.scielo.br/scielo.php?script=sci_arttext\&pid=S010373312016000200525\&lng=en. $\quad$ DOI: https://doi. org/10.1590/S0103-73312016000200010

23. Mendes HJA. A relação entre os cirurgiões-dentistas e as operadoras de planos de Saúde no município de Bauru-SP [dissertação] [Internet]. Bauru: Faculdade de Odontologia de Bauru, Universidade de São Paulo; 2005. [Acesso em 2019 nov. 10]. Disponível em: https://teses.usp.br/teses/ disponiveis/25/25141/tde-16082005-094702/es.php

24. Brasil. Presidência da República, Subchefia para Assuntos Jurídicos. Constituição da República Federativa do Brasil de 1988. Diário Oficial da República Federativa do Brasil. 1988 out. 05 ; Seção 1. p. 1-32.

25. Brasil. Presidência da República, Subchefia para Assuntos Jurídicos. Lei no 8.080, de 19 de setembro de 1990. Dispõe sobre as condições para a promoção, proteção e recuperação da saúde, a organização e o funcionamento dos serviços correspondentes e dá outras providências. Diário Oficial da União. 1990 set. 20; Seção 1. p. 1.

26. Brasil. Presidência da República, Subchefia para Assuntos Jurídicos. Lei no 8.689, de 27 de julho de 1993. Dispõe sobre a extinção do Instituto Nacional de Assistência Médica da Previdência Social (Inamps) e dá outras providências. Diário Oficial da União. 1993 jul. 28; Seção 1. p. 1-2.

27. Brasil. Ministério da Saúde. Portaria n ${ }^{\circ} 545$, de 20 de maio de 1993. Estabelece normas e procedimentos reguladores do processo de descentralização da gestão das ações e serviços de saúde, através da Norma Operacional Básica - SUS 01/93 [Internet]. Diário Oficial da União. 1993 mai. 20 [Acesso em 2019 dez. 19]. Disponível em: http://siops.datasus.gov. br/Documentacao/Portaria\%20545_20_05_1993.pdf

28. Brasil. Ministério da Saúde. Portaria $n^{\circ} 2.203$, de 5 de novembro de 1996. Aprova a norma operacional básica do Sistema Único de Saúde NOB-SUS 01/96. Diário Oficial da União. 1996 nov. 05; Seção1. p. 22932-22940.

29. Brasil. Ministério da Saúde. Agência Nacional de Saúde Suplementar (ANS). Caderno de Informações da Saúde Suplementar: beneficiários, operadoras e planos. Rio de Janeiro: ANS; 2007.

30. Pereira AS, Neumann DG, Caetano JC, Finkler M, Mussi DS. Satisfação de usuários de planos odontológicos: um estudo de caso em uma operadora de autogestão. RFO [Internet]. 2016 [Acesso em 2019 nov. 5];21(1):49-54. Disponível em: http://seer.upf.br/index.php/rfo/article/ view/5526. DOI: https://doi.org/10.5335/rfo.v21i1.5526

31. Brasil. Presidência da República, Subchefia para Assuntos Jurídicos. Lei no 8.078, de 11 de setembro de 1990. Dispõe sobre a proteção do consumidor e dá outras providências [Internet]. Brasília, DF; 1990. [Acesso em 2019 nov. 2]. 
Feitosa LBL, Santos DR, Santos RMC. Conflitos na relação entre Cirurgiões-dentistas e operadoras de Odontologia suplementar.

Disponível em: https://www.planalto.gov.br/ccivil 03/leis/ 18078.htm

32. Brasil. Presidência da República, Subchefia para Assuntos Jurídicos. Decreto $\mathrm{n}^{\circ}$ 2.181, de 20 de março de 1997. Dispõe sobre a organização do Sistema Nacional de Defesa do Consumidor - SNDC, estabelece as normas gerais de aplicação das sanções administrativas previstas na Lei $\mathrm{n}^{\circ}$ 8.078 , de 11 de setembro de 1990, revoga o Decreto $\mathrm{N}^{\circ}$ 861, de 9 julho de 1993, e dá outras providências [Internet]. Brasília, DF; 1993. [Acesso em 2019 nov. 2]. Disponível em: http://www.planalto.gov.br/ccivil_03/decreto/d2181. htm

33. Pietrobon L, Silva CM, Batista LRV, Caetano JC. Health care plans: interfaces between the public and private system in the dental sector. Ciênc Saúde Coletiva [Internet]. 2008 [Acesso em 2019 dez. 10];13(5):1589-99. Disponível em: $\quad$ http://www.scielo.br/scielo.php?script=sci arttext\&pid=S1413-81232008000500023\&lng=en. DOI: http://dx.doi.org/10.1590/S1413-81232008000500023

34. Brasil. Presidência da República, Subchefia para Assuntos Jurídicos. Lei $n^{0}$ 9.656, de 3 de junho de 1998. Dispõe sobre os planos e seguros privados de assistência à saúde [Internet]. Brasília, DF; 1998. [Acesso em 2019 nov. 2]. Disponível em: http://www.planalto.gov.br/ccivil_03/leis/ 19656.htm

35. Brasil. Presidência da República, Subchefia para Assuntos Jurídicos. Lei n ${ }^{\circ}$ 9.961, de 28 de janeiro de 2000. Cria a Agência Nacional de Saúde Suplementar - ANS e dá outras providências [Internet]. Brasília, DF; 2000. [Acesso em 2019 nov. 2]. Disponível em: http://www.planalto.gov.br/ ccivil_03/Leis/L9961.htm

36. Brasil. Ministério da Saúde. Conselho Nacional de Secretários de Saúde (CONASS). Sistema Único de Saúde. Brasília: CONASS; 2011.

37. Brasil. Ministério da Saúde. Agência Nacional de Saúde Suplementar (ANS). Caderno de Informações da Saúde Suplementar: beneficiários, operadoras e planos. Rio de Janeiro: ANS; 2006.

38. Brasil. Ministério da Saúde. Agência Nacional de Saúde Suplementar (ANS). Caderno de Informações da Saúde Suplementar: beneficiários, operadoras e planos. Rio de Janeiro: ANS; 2011.

39. Brasil. Ministério da Saúde. Agência Nacional de Saúde Suplementar (ANS). Caderno de Informações da Saúde Suplementar: beneficiários, operadoras e planos. Rio de Janeiro: ANS; 2016.

40. Narvai PC. Saúde bucal coletiva: caminhos da odontologia sanitária à bucalidade. Rev Saúde Pública [Internet]. 2006 [Acesso em 2019 dez. 10];40(spe):141-147. Disponível em: $\quad$ http://www.scielo.br/scielo.php?script=sci arttext\&pid=S0034-89102006000400019\&lng=en. DOI: http://dx.doi.org/10.1590/S0034-89102006000400019

41. Brasil. Ministério do Trabalho e Emprego. Escola Nacional da Inspeção do Trabalho (ENIT). Normas Regulamentadoras de Segurança e Saúde do Trabalhador [Internet]. Brasília: Ministério do Trabalho e Emprego; 2003. [Acesso em 2019 dez. 10]. Disponível em: https://enit.trabalho.gov.br/portal/ index.php/seguranca-e-saude-no-trabalho/sst-menu/sst- normatizacao/sst-nr-portugues?view=default

42. Lima CA. Pesquisa de satisfação dos dentistas sobre o atendimento a planos de saúde. Jornal do CROBA. 2002;45:6-7.

43. Garcia PPNS, Cobra CS. Condições de trabalho e satisfação de cirurgiões-dentistas credenciados por convênios odontológicos. Rev Odontol UNESP. 2004;33(3):115-22.

44. Vilela MC, Silva MVSC, Barbieri AA, Orenha ES, Naressi SCM. Perfil e grau de satisfação profissional de cirurgiõesdentistas credenciados a uma operadora de planos odontológicos. Braz Dent Sci. 2010;13(6):39-44. DOI: https://doi.org/10.14295/bds.2010.v13i1/2.12

45. Federação Interestadual dos Odontologistas (FIO), Conselho Federal De Odontologia (CFO), Associação Brasileira de Odontologia (ABO), Federação Nacional dos Odontologistas (FNO). Resultado da pesquisa sobre o perfil do cirurgião-dentista no Brasil [Internet]. Brasília, DF; 2003. [Acesso em: 2019 set. 26]. Disponível em: http:// www.crors.org.br

46. Bragança DPP. Auditoria odontológica: aspectos relacionados ao gerenciamento de dados e custos operacionais [dissertação] [Internet]. Piracicaba: Faculdade de Odontologia de Piracicaba, Universidade Estadual de Campinas; 2010. [Acesso em 2019 dez. 19]. Disponível em: http://repositorio.unicamp. br/bitstream/REPOSIP/290737/1/Braganca DanielPereiraParreirasde_M.pdf

47. Moraes DA, Maluf F, Tauil PL, Portillo JAC. Considerações bioéticas sobre o processo de trabalho do cirurgião dentista na saúde suplementar. Comun Ciênc Saúde. 2016;27(4):267-78.

48. Paixão HH. A odontologia sob o capital: o mercado de trabalho e a formação universitário-profissional do cirurgião-dentista [dissertação]. Belo Horizonte: Faculdade de Educação, Universidade Federal de Minas Gerais; 1979.

49. Bleicher L. Autonomia ou assalariamento precário? O trabalho dos cirurgiões-dentistas na cidade de Salvador [tese] [Internet]. Salvador: Faculdade de Filosofia e Ciências Humanas, Universidade Federal da Bahia; 2011. [Acesso em 2019 dez. 20]. Disponível em: http://repositorio.ufba. br/ri/handle/ri/19619

50. Noronha FM, Salles DMT. A ferramenta auditoria aplicada à odontologia. In: Anais do Congresso Brasileiro de Administração, 2004.

51. Schiavolin NL. Convênio e cooperativas odontológicas na região metropolitana de São Paulo: uma análise operacional [dissertação] [Internet]. Piracicaba: Faculdade de Odontologia de Piracicaba, Universidade Estadual de Campinas; 2005. [Acesso em 2019 dez. 19]. Disponível em: http://www.repositorio.unicamp.br/handle/ REPOSIP/289889

52. Brasil. Conselho Federal de Odontologia. Resolução CFO $\mathrm{n}^{\circ} 118$, de 11 de maio de 2012. Revoga o Código de Ética Odontológica aprovado pela Resolução CFO 42/2003 e aprova outro em substituição. Diário Oficial da União. 2012 jun. 14; Seção 1. p. 118.

53. Alves M. Planos odontológicos crescem [Internet]. São Paulo; 2016. [Acesso em 2020 jul. 10]. Disponível 
Feitosa LBL, Santos DR, Santos RMC. Conflitos na relação entre Cirurgiões-dentistas e operadoras de Odontologia suplementar.

em: http://www.sinog.com.br/portal/impressao-noticia. php?noticia $=186$

54. Montenegro FLB. Convênios odontológicos: uma saída para a crise? JADA- Brasil. 1999;2:79-80.

55. Rodrigues RCM. A odontologia suplementar no Distrito Federal [dissertação] [Internet]. Brasília: Faculdade de Ciências da Saúde, Universidade de Brasília; 2002. [Acesso em 2019 dez. 19]. Disponível em: https://repositorio.unb. $\mathrm{br} /$ handle/10482/34683

Recebido em: 14/11/2019

Aceito em: 18/03/2020
56. Merhy E, Júnior HM. Regulação pública da assistência na saúde suplementar a quem interessa? Jornal do Conselho Regional de Medicina de Minas Gerais. 2001;24(3):705-14.

57. Araújo MF, Correia CL, Groisman S, Toledo E. Convênios odontológicos: visão dos cirurgiões-dentistas do Município de Duque de Caxias/RJ. Rev Bras Odontol [Internet]. 2012 [Acesso em 2019 dez. 19];69(2):165-9. Disponível em: http://revista.aborj.org.br/index.php/rbo/article/view/425. DOI: http://dx.doi.org/10.18363/rbo.v69n2.p.165 